\title{
interernoive 2021
}

\section{Soundscape: \\ Progress in the past 50 years and challenges in the next 50 years}

\author{
Jian Kang ${ }^{1}$ \\ UCL Institute for Environmental Design and Engineering, The Bartlett \\ University College London, London WC1H 0NN \\ United Kingdom
}

\begin{abstract}
Soundscape is defined by the ISO as the 'acoustic environment as perceived or experienced and/or understood by a person or people, in context'. Different from conventional noise control engineering, soundscape promotes a holistic approach, regarding sounds as 'resources' rather than just 'wastes'. This paper first briefly reviews the soundscape progress in the past 50 years, showing that there has been a recognised focus shift from noise control to soundscape creation, and also a step change from soundscape concept to practice. Then the current developments and needs in soundscape are discussed in terms of soundscape understating and exchanging, collecting and documenting, harmonising and standardising, creating and designing, and outreaching, showing that while considerable works have been carried out, much work is still needed, in terms of basic research, and more importantly, research towards practice. Finally, some major challenges in the next 50 years are explored, considering the new industrial revolution, climate change and changing living styles.
\end{abstract}

\section{INTRODUCTION}

Soundscape has brought a step change in the field of environmental acoustics, beyond noise control engineering, by better integrating human perception and considering both wanted and unwanted sounds. This paper first briefly reviews the progress in the past 50 years in soundscape, and then it focuses on the current developments in various facets, from basic research, to practice, to outreaching [1-5]. Finally, it discusses the future challenges it will face in the next 50 years.

\section{PROGRESS IN THE PAST 50 YEARS: FROM CONCEPT TO PRACTICE}

\subsection{Pioneering Work in Soundscape}

Just over 50 years ago, pioneering works in soundscape were carried out by Schafer: a musician and composer, his early soundscape work had always been about relationship between the ear, human beings, sound environments and society [6].

In 1975, Schafer led a group on a European tour of five villages where they made detailed investigations of the soundscape. Later the five villages were revisited to undertake comparative studies, to analyse how their soundscapes had changed due to urbanisation [7-8].

\subsection{The World Forum for Acoustic Ecology}

The World Forum for Acoustic Ecology (WFAE) was founded in 1993, with members who share a common concern with the state of the world soundscape as an ecologically balanced entity. The WFAE also has its annual, peer-reviewed journal, <Soundscape: The Journal of Acoustic Ecology $>$. While the WFAE represents a multi-disciplinary spectrum of individuals engaged in the study of the social, cultural, and ecological aspects of the sonic environment, less attention has been paid to the planning, designing and engineering the soundscapes of built environments.

\footnotetext{
${ }^{1}$ j.kang@ucl.ac.uk
} 


\subsection{From Concept to Practice}

In 2002, the publication of the EU Directive Relating to the Assessment and Management of Environmental Noise (END) [9] led to a number of major actions, including preserving quiet areas. However, it was recognised that reducing sound level is not always feasible and cost-effective, and more importantly, will not necessarily lead to improved quality of life, as people's acoustic comfort evaluation is also related to the sound type, the user characteristics and other factors [10-11]. Indeed, it was shown that the environmental noise annoyance only depends on approximately $30 \%$ of the physical facets of parameters such as acoustic energy $[1,12]$. While it was not clear how to determine those quiet areas, where to go with it, how to use it, or how to incorporate it in design, a step change was needed, by developing a new method to assess sound environment quality.

Correspondingly, ISO/TC43/SC1/Working Group 54 was formed to work on 'Perceptual assessment of soundscape quality'. In ISO 12913-1:2014 Acoustics - Soundscape - Part 1: Definition and conceptual framework, soundscape is defined as the 'acoustic environment as perceived or experienced and/or understood by a person or people, in context' [13].

In other words, 'soundscape' is different from 'acoustic environment' as it relates to perceptual constructs rather than just physical phenomena. It promotes a holistic approach, regarding sounds as 'resources' rather than just 'wastes', and focuses on "wanted" (preference) rather than just "unwanted" (discomfort) sounds. While soundscape has always been related to many disciplines including acoustics, aesthetics, anthropology, architecture, ecology, ethnology, communication, design, human geography, information, landscape, law, linguistics, literature, media arts, medicine, musicology, noise control engineering, philosophy, pedagogics, psychology, political science, religious studies, sociology, technology and urban planning, with the EU Directive END as a key driver, soundscape creation attracted much attention of many acousticians, engineers, designers and planners, moving from soundscape concept to practice.

The impacts of soundscape include: (1) Health: It can help to provide supportive environments which prevent the degradation of functional health, and enhance the engagement in health promoting activities less likely in unpleasant neighbourhoods. (2) Culture: It is important in terms of 'sensing of places', tourism, and conservation. (3) Economy: It can bring prosperity to the economy, i.e. property prices, offset health costs through provision of restorative living spaces, reduce costs caused by antisocial behaviours, and prevent unnecessary noise mitigating actions [14,15].

Soundscape activities have since increased significantly, as reflected in the number of publications shown in Figure 1. There have been increasing special sessions in conferences in the field of acoustics such as Internoise, euronoise, ICA, ICSV, ICBEN, and Westpac, and also in the field of planning such as AESOP. There have also been increasing national and international research projects, practical projects [15], as well as networks such as the EU-COST network on Soundscape of European Cities and Landscapes [15], and the Global Sustainable Soundscape Network GSSN.

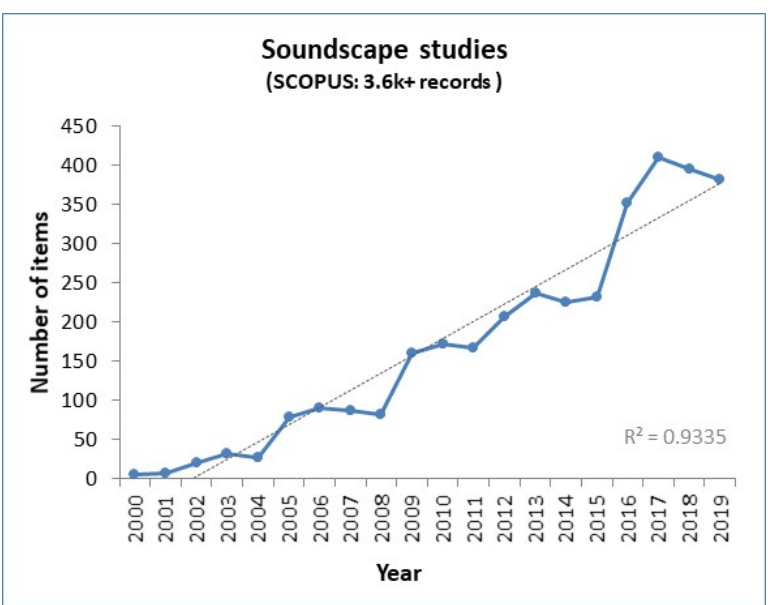

Figure 1: Number of soundscape studies published since 2000 as covered in Scopus database. TITLE-ABS-KEY(soundscape) AND ( LIMIT-TO ( SUBJAREA,"PHYS" ) OR LIMIT-TO ( SUBJAREA,"ARTS" ) OR LIMIT-TO ( SUBJAREA,"SOCI" ) OR LIMIT-TO ( SUBJAREA,"COMP" ) OR LIMIT-TO ( SUBJAREA,"ENVI" ) OR LIMIT-TO ( SUBJAREA,"ENGI" ) OR LIMIT-TO ( SUBJAREA,"EART" ) OR LIMIT-TO ( SUBJAREA,"PSYC" ) OR LIMIT-TO ( SUBJAREA,"MULT") OR LIMIT-TO ( SUBJAREA,"NEUR" ) OR LIMIT-TO ( SUBJAREA,"ENER" ) OR LIMIT-TO ( SUBJAREA,"BUSI" ) OR LIMIT-TO ( SUBJAREA,"DECI" ) OR LIMIT-TO ( SUBJAREA,"HEAL" ) OR LIMIT-TO ( SUBJAREA,"NURS" ) OR LIMIT-TO ( SUBJAREA,"ECON" ) ) AND ( LIMIT-TO ( PUBYEAR,2000-2020) 


\section{CURRENT DEVELOPMENTS}

To review the current developments of soundscape research and practice, a framework is used, where five main issues are considered, as shown in Figure 2. The framework is based on the EU-COST network on Soundscape of European Cities and Landscapes (http://soundscape-cost.org/), with partner organisations from 23 COST countries and 7 outside Europe, covering a range of disciplines in science, engineering, social science, humanity and medicine [15]. In this section, works in those facets are reviewed, using the work from the author's research teams as examples.

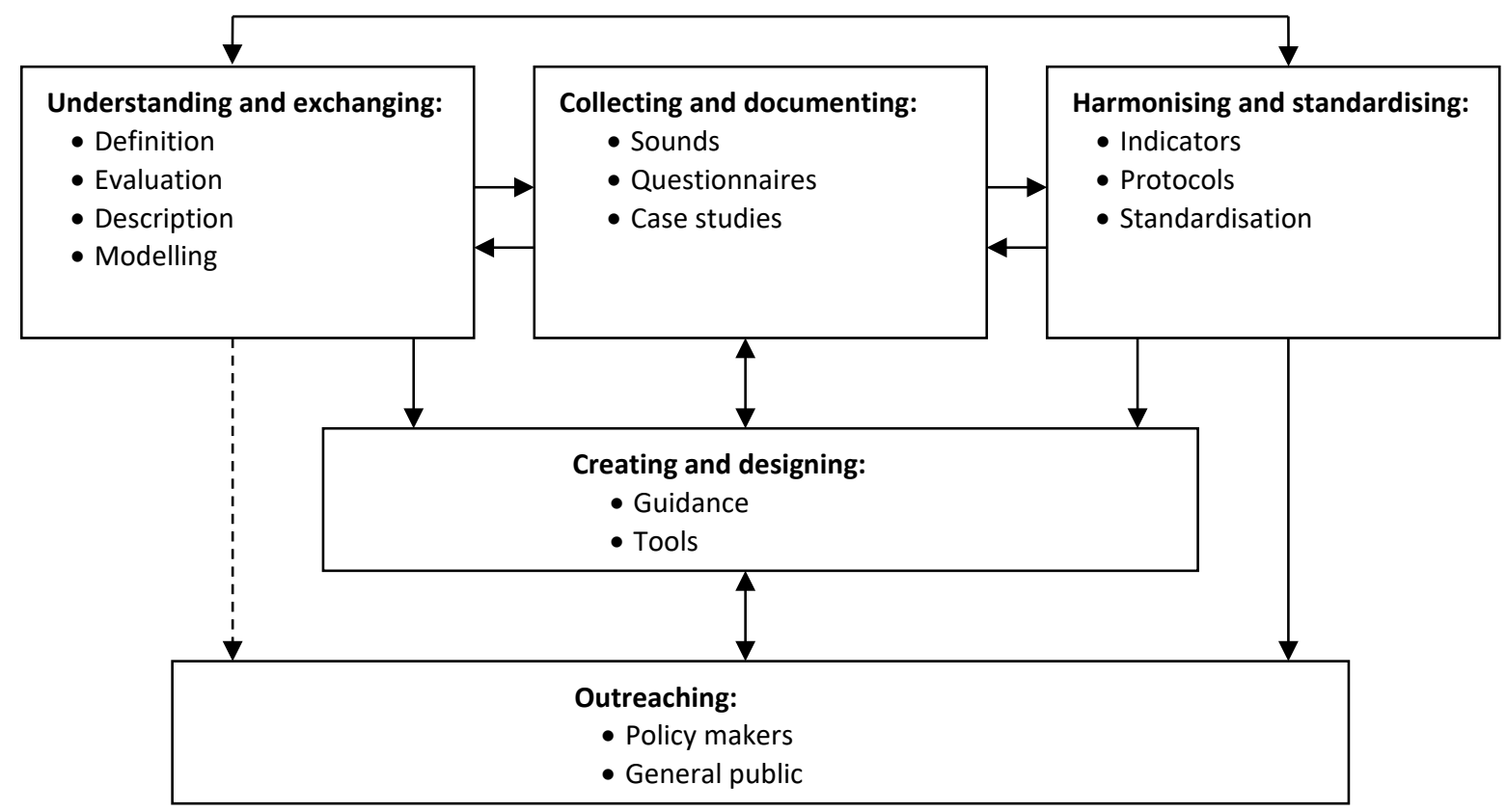

Figure 2: A framework for soundscape research and practice [15].

\subsection{Understanding and Exchanging}

\subsubsection{Definition}

To understand the elements in the perceptual construct of soundscape, the ISO/TC43/SC1/Working Group 54 proposed a framework including interactions among context, sound sources, acoustic environment, auditory sensation, interpretation of auditory sensation, responses, and outcomes [13].

\subsubsection{Evaluation}

In terms of psychological or subjective evaluation of soundscape, a large amount of investigations have been carried out, considering (1) a range of spaces and functions including urban streets, urban open public spaces, parks, schools, bus stations, theme streets, cycle paths, outdoor concerts, racing tracks, archaeological sites, covered spaces, underground shopping streets; (2) a range of sound sources, from noise sources including industrial noises, aircraft noises, road noises, wind turbines, amplified music; to positive sounds including natural sounds; to sources with mixed perceptions such as an infant's cry; and (3) a range of users, where their social and demographical characteristics have been considered for various users, also for specific groups such as children, deaf, hearing impaired and blind people $[1,14]$.

In terms of the physiological side of soundscape, there has been relatively limited, but increasing work, considering a range of indicators including heart rate, respiratory rate and forehead electromyography levels, and using a range of tools including fMRI techniques [16-17]. Some initial relations have been established between psychological, physiological, and health indicators [18-19].

A wide range of perspectives have been explored in soundscape, including how attention on the external environment is shaped internally; interactions between emotion and soundscape; how expectation affects soundscape; interactions between behaviour and soundscape; how social relations, integration and support affect soundscape; how meaning is attached to the objects within a cultural/societal context; the effect that a stressed or harmonised human-environment relationship can have on mental health; and soundscape valuation, following noise valuation [20-24]. 
Multi-sensory interactions have also been explored and demonstrated, including audio-visual interactions, sound-smell interactions, and sound-thermal interactions [25-27].

\subsubsection{Description}

Based on the above evaluation work, determining essential factors and framework for soundscape description is important for understanding as well as creating/designing soundscapes. A framework of designable factors for soundscape in urban open public spaces has been developed [28]. A taxonomy has also been developed showing categories of places, sounds, and sound sources [29].

\subsubsection{Modelling}

It is important to integrate the knowledge acquired from different fields into explicit modelling. Using the data obtained from a large-scale survey, a model based on artificial neural networks has been developed to predict soundscape perception [30]. More fundamentally, a bottom-up approach is also needed, considering the individual sensory, cognitive and emotional mechanisms. Modelling physics side, namely sound propagation is a space is also relevant here, where a number of models have been developed [31].

\subsection{Collecting and Documenting}

Gathering and maintaining a repository of soundscape data is important, to be achieved and re-analysed and studied from inter- and trans- disciplinary perspectives. In terms of sound sources, there have been a considerable number of databases of various kinds, using different recording techniques. While those are useful for soundscape studies, more relevant databases would be those with context information, such as visual information. However, such databases are still limited although in recent years there is an increasing number, such as those based on the 'Urban Soundscapes of the World' [32], European Research Council (ERC) SSID (Soundscape Indices) project [33], and SONYC Sounds of New York City project [34].

Given that a large number of soundscape questionnaire surveys and interviews have been carried out, such as those based on RUROS [35] and SSID projects [33], with 10,000 and 4000 field surveys/interviews worldwide respectively, there is a recognised need to create coordinated and comparable databases.

To move from research to practice, a collection of good soundscape design examples and case studies is vital across sectors including researchers, practitioners and policy makers. There have been some initial efforts [14] and a more systematic collection project is on-going [36].

\subsection{Harmonising and Standardising}

While it is argued that standardisation could restrict the creativity in designing soundscapes, from planning viewpoint, it is useful to have standards. The ISO/TC43/SC1/Working Group 54 has generated a series of standards and technical specifications on perceptual assessment of soundscape quality, including ISO 12913-1: 2014: Part 1: Definition and conceptual framework [13], ISO/TS 12913-2: 2018: Part 2: Data collection and reporting requirement [37], and ISO/TS 12913-3: 2019: Part 3: Data analysis [38]. Currently the Working Group is developing ISO/TS 12913-4 - Part 4: Design and intervention.

Correspondingly, a number of more detailed and specific protocols have been developed, such as those for soundscape description and evaluation, considering cross-cultural and cross-contextual differences; and measurement procedures with respect to a balance between scientific accuracy and practical applicability, also considering comparability and reproducibility. A recent example is the SSID protocol [39].

There is still a recognised need to develop a new set of indicators to characterise sound quality of environments that improves significantly on the conventional decibel level approach that has been the basis of current regulations worldwide for decades. The indicators should be suitable to assess health related quality of life and functional health which can then be used to evaluate claims related to health-promotion benefits. Efforts have been made to develop indicators including fuzzy noise limits, tranquillity rating, speech intelligibility, similarity index, and hierarchical clustering [2-4].

An ongoing ERC Advanced Grant project aims to establish "soundscape indices" (SSID) [33]. By taking psychological, (psycho)acoustical, neural and physiological, and contextual factors into 
account, SSID will adequately reflect levels of human comfort, to integrate side-by-side with (and eventually replace) decibel-based metrics into existing (international) regulations. Steps to achieve this include:

(1) to characterise soundscapes, by capturing acoustic environments and establishing a comprehensive database;

(2) to identify key factors and their influence on soundscape quality based on the database, by conducting laboratory psychological evaluations, acoustical/psychoacoustic factors analysis, and also, to research the neural and psychophysiological underpinnings of soundscape experience;

(3) to develop, test and validate the soundscape indices, by analysing the influences of various factors, and by developing prediction models.

The soundscape indices may take the form of a single index or a set of indices. For the former, it could be SSID $=\mathrm{f}($ physical factors $)+\mathrm{f}($ contextual factors $)+\ldots$, with corrections by socio-demographical factors and modifications with psychological, neural, and physiological considerations. For the latter, the SSID will reflect multiple attributes, and in the same time, they could also be regarded as intermediate indices.

\subsection{Creating and Designing}

In the process of applying soundscape research into practice, there is a need for practical guidance in soundscape design. Such guidance [40], which currently is still limited, should include design processes, effectiveness of design changes, as well as good practical examples. In the design process, a key component is public participation [41]. It is also of significance to provide guidelines for the preservation of architectural heritage sites from a soundscape perspective.

Correspondingly, soundscape tools and software are also essential, considering different stages and scales, including planning, designing, and management/operation. A number of mapping tools have been developed, such as soundscape perception prediction model based on ANN [30], and sound source mapping tools [42-43]. A soundscape management model for delivery sound environment has also been developed, where with the input in contextual and acoustic factors, the model can predict soundscape quality [44].

Auralisation tools are especially relevant and important for soundscape design and also for public participation. While such tools have been developed well in room acoustics, for environmental soundscapes, there are many challenges, including multiple, complex, and moving sources, under complicated situations, as well as speed needed to generate results. On the other hand, there are relatively low requirements in terms of accuracy compared to room acoustics. Therefore, it is important to explore simplifications through subjective experiments [45-46].

\subsection{Outreaching}

As discussed above, policies have been a major driver to move from research to practice. For policy makers it is important to demonstrate benefits, especially health effects, and provide successful examples and tools. With the influence of soundscape work, the UK noise policy [47] has been moved from noise mitigation to noise management, and another step change was that in 2018, the Welsh Government published its Noise and Soundscape Action Plan [48]. Soundscape is also relevant to a wider range of policy. For example, it should be recognised that soundscape studies are not only for the improvement of the current sound environment but also for the conservation of our sound environments which can be classified as acoustic heritages [15].

It is equally important to create awareness amongst the general public, especially given that soundscape is relevant to a much wider range of citizens than noise. For that soundscape art installations could be effective [49], as well as apps which involve participation of the general public [50].

\section{CHALLENGES IN THE NEXT 50 YEARS}

In the next 50 years there will be a range of major changes in various aspects of our life and environment. Correspondingly, this section tries to briefly explore long-term challenges in soundscape research and practice in such changing situations, although for immediate or short-term future developments, some discussions have also been made in Section 3. 


\subsection{New Industrial Revolution}

New technologies are being developed rapidly and our cities and living environments are becoming smarter, with many devices with sensors rapidly increasing in popularity [51]. This could lead to smarter and adjustable soundscapes, in terms of space and time domain, integrating more the needs of specific users.

With the development of electric vehicles, our sound environments will become quieter. However, it is noted that during the COVID-19 lock-down, although the environmental noise levels were much lower [52], there was a sharp increase in noise complaints, as some other sound sources became more annoying even their sound levels were unchanged [53]. This again demonstrated the needs and challenges in soundscape creation, compared to merely engineering noise control.

\subsection{Climate Change}

With the tendency of climate change and global warming, there will be a range of changes in our environmental conditions, and this in turn, will bring changes in biodiversity, urban morphology, as well as cultural changes. Those will affect sound sources, sound propagation, and sound preferences. For example, it has been shown that for sounds from water features, surrounding speech, and birdsongs, the sound preferences differ significantly with different climate and culture conditions [1,54].

\subsection{Changing Living Style}

Along with the new industrial revolution and climate change, people's living style will also change. For example, people may work from home more, and use public spaces in a different way. Correspondingly, the soundscape evaluation framework would also change. It has been shown that for indoor soundscape in residential environments, there is a different assessment mode compared to that in urban open public spaces [55].

\section{CONCLUSIONS}

Started from the pioneering work in soundscape just over 50 years ago, in the field of environmental acoustics there has been a focus shift from noise control to soundscape creation, and also a shift from soundscape concept to practice. While considerable works have been carried out in terms of soundscape understating and exchanging, collecting and documenting, harmonising and standardising, creating and designing, and outreaching, much work is still needed, in terms of research towards practice, as well as basic research. In the next 50 years, there will be some major challenges along with new industrial revolution, climate change and changing living styles.

\section{ACKNOWLEDGEMENTS}

The author is grateful to all his previous and current team members, as well as collaborators, especially the participants of the COST TUD Action TD-0804, for beneficial discussions. This work received funding from the European Research Council (ERC) under the European Union's Horizon 2020 research and innovation programme (ERC Advanced Grant No. 740696).

\section{REFERENCES}

1. Kang, J. Urban Sound Environment, Taylor \& Francis incorporating Spon, London, 2007.

2. Kang, J. Soundscapes: where are we? Proceedings of the Institute of Acoustics \& Belgium Acoustical Society 32 (Pt 3), 1-18 (2010).

3. Kang, J. From dBA to soundscape indices: managing our sound environment. Frontiers of Engineering Management, 4, 184-192 (2017).

4. Kang, J. Urban sound planning - A soundscape approach. Proceedings of the Acoustics 2019, Melbourne, Australia, 2019.

5. Kang, J., Aletta, F., Gjestland, T., Brown, L., Botteldooren, D., Schulte-Fortkamp, B., Lercher, P., van Kamp, I., Genuit, K., Fiebig, A., Bento Coelho, J., Maffei, L. \& Lavia, L. Ten questions on the soundscapes of the built environment. Building and Environment, 108, 284-294 (2016).

6. Schafer, R. M. The Tuning of the World, Knopf, New York, 1977, republished, as Our Sonic Environment and the Soundscape: The Tuning of the World, Destiny Books, Vermont, 1994. 
7. Järviluoma, H. Acoustic environments in change: five village soundscapes revisited. Soundscape: The Journal of Acoustic Ecology, 1, 25 (2000).

8. Järviluoma, H., Kytö, M., Truax, B., Uimonen, H., Vikman, N. \& Schafer, R. M. Acoustic Environments in Change \& Five Village Soundscapes, TAMK University of Applied Sciences, 2010.

9. EU (European Union). Directive of the European Parliament and of the Council of Relating to the Assessment and Management of Environmental Noise, 2002.

10. Yang, W. \& Kang, J. Soundscape and sound preferences in urban squares: A case study in Sheffield. Journal of Urban Design, 10, 69-88 (2005).

11. Yang, W. \& Kang, J. Acoustic comfort evaluation in urban open public spaces. Applied Acoustics, 66, 211-229 (2005).

12. Guski, R. Psychological methods for evaluating sound quality and assessing acoustic information. Acustica united with acta acustica, 83, 765-774 (1997).

13. International Organization for Standardization. ISO 12913-1:2014 Acoustics - Soundscape - Part 1: Definition and Conceptual Framework, Geneva, 2014.

14. Kang, J. \& Schulte-Fortkamp, B. (ed.), Soundscape and the Built Environment, Taylor \& Francis incorporating Spon, London, 2016.

15. Kang, J., Chourmouziadou, K., Sakantamis, K, Wang, B. \& Hao, Y. (ed.) Soundscape of European Cities and Landscapes. EU COST, Oxford, 2013.

16. Hunter, M. D., Eickhoff, S. B., Pheasant, R. J., Douglas, M. J., Watts, G. R., Farrow, T. F. D., Hyland, D., Kang, J., Wilkinson, I. D., Horoshenkov, K. V. \& Woodruff, P. W. R. The state of tranquility: Subjective perception is shaped by contextual modulation of auditory connectivity. NeuroImage, 53, 611-618 (2010).

17. Li, Z. \& Kang, J. Sensitivity analysis of changes in human physiological indicators observed in soundscapes. Landscape and Urban Planning, 190, 103593 (2019).

18. Erfanian, M., Mitchell, A., Kang, J. \& Aletta, F. The psychophysiological implications of soundscape: A systematic review of empirical literature and a research agenda. Int. J. Environ. Res. Public Health, 16, 3533, 1-20 (2019).

19. Aletta, F., Oberman, T. \& Kang, J. Associations between positive health-related effects and soundscapes perceptual constructs: A systematic review. International Journal of Environmental Research and Public Health, 15, 2392, 1-15 (2018).

20. Qin, Y., Zhao, W. \& Kang, J. Effects of background sound sources types on emotion and activities in activity spaces of elderly care facilities. Journal of Human Settlements in West China, 35, 43-49 (2020) (in Chinese).

21. Ren, X., Kang, J., Zhu, P. \& Wang, S. Soundscape expectations of rural tourism: A comparison between Chinese and English potential tourists. Journal of the Acoustical Society of America, 143, 373377 (2018).

22. Aletta, F., Lepore, F., Kostara-Konstantinou, E., Kang, J. \& Astolfi, A. An experimental study on the influence of soundscapes on people's behaviour in an open public space. Applied Sciences, 6, 276, 112 (2016).

23. Jiang, L., Bristow, A., Kang, J., Aletta, F., Nellthorp, J., Thomas, A., Notley, H. \& Thomas, R. From noise valuation to soundscape valuation. Euronoise 2021.

24. Cao, J. \& Kang, J. Social relationships and patterns of use in urban public spaces in China and the United Kingdom. Cities, 93, 188-196 (2019).

25. Ren, X. \& Kang J. Interactions between landscape elements and tranquillity evaluation based on eye tracking experiments. Journal of the Acoustical Society of America, 138, 3019-3022 (2015).

26. Ba, M. \& Kang, J. Effect of a fragrant tree on the perception of traffic noise. Building and Environment, 156, 147-155 (2019).

27. Jin, Y., Jin, H. \& Kang, J. Effects of sound types and sound levels on subjective environmental evaluations in different seasons. Building and Environment, 183, 107215 (2020).

28. Zhang, M. \& Kang, J. Towards the evaluation, description, and creation of soundscape in urban open spaces. Environment and Planning B: Planning and Design, 34, 68-86 (2007).

29. Brown, A. L., Kang, J. \& Gjestland, T. Towards standardization in soundscape preference assessment. Applied Acoustics, 72, 387-392 (2011). 
30. Yu, L. \& Kang, J. Modeling subjective evaluation of soundscape quality in urban open spaces - An artificial neural network approach. Journal of the Acoustical Society of America, 126, 1163-1174 (2009).

31. Kang, J. Numerical modelling of the sound fields in urban squares. Journal of the Acoustical Society of America, 117, 3695-3706 (2005).

32. http://urban-soundscapes.org/ (accessed 01.05.2021)

33. Kang, J., Aletta, F., Oberman, T., Erfanian, M., Kachlicka, M., Lionello, M. \& Mitchell, A. Towards soundscape indices. Proceedings of 23rd International Congress on Acoustics, integrating 4th EAA Euroregio 2019, pp.2488-2495, Aachen, Germany, 2019.

34. https://wp.nyu.edu/sonyc/(accessed 01.05.2021)

35. Yu, L. \& Kang, J. Effects of social, demographic and behavioral factors on sound level evaluation in urban open spaces. Journal of the Acoustical Society of America, 123, 772-783 (2008).

36. https://www.soundscape-intervention.org/(accessed 01.05.2021)

37. International Organization for Standardization. ISO 12913-2:2018 Acoustics - Soundscape - Part 2: Data Collection and Reporting Requirement, Geneva, 2018.

38. International Organization for Standardization. ISO 12913-3:2019 Acoustics - Soundscape - Part 3: Data Analysis, Geneva, 2019.

39. Mitchell, A., Oberman, T., Aletta, F., Erfanian, M., Kachlicka, M., Lionello, M. \& Kang, J. The Soundscape Indices (SSID) Protocol: A method for urban soundscape surveys-Questionnaires with acoustical and contextual information. Applied Sciences, 10, 2397, 1-27 (2020).

40. Kang, J., Yang W. \& Zhang M. Sound environment and acoustic comfort in urban spaces. In: Nikolopoulou, M. (ed.), Designing Open Spaces in the Urban Environment: a Bioclimatic Approach, Jointly published by the European Commission and the Centre for Renewable Energy Sources, Chapter 7, pp32-36, 2004.

41. Xiao, J., Lavia, L. \& Kang, J. Towards a participatory urban soundscape planning framework. Journal of Environmental Planning and Management, 61, 677-698 (2017).

42. Hao, Y., Kang, J. \& Krijnders, J. D. Integrated effects of urban morphology on birdsong loudness and visibility of green areas. Landscape and Urban Planning, 137, 149-162 (2015).

43. Liu, J., Kang, J., Behm, H. \& Luo, T. Effects of landscape on soundscape perception: soundwalks in city parks. Landscape and Urban Planning, 123, 30-40 (2014).

44. Kang, J., Hao, Y., Yang, M. \& Lavia, L. Soundscape evaluation and indicators for delivery sound environment. Proceedings of the 22nd International Congress on Sound and Vibration (ICSV), Florence, Italy, 2015.

45. Smyrnova, Y. \& Kang, J. Determination of perceptual auditory attributes for the auralization of urban soundscapes. Noise Control Engineering Journal, 58, 508-523 (2010).

46. Xu, C. \& Kang, J. Soundscape evaluation: binaural or monaural? Journal of the Acoustical Society of America, 145, 3208-3217 (2019).

47. https://assets.publishing.service.gov.uk/government/uploads/system/uploads/attachment data/file/69533/pb13750-noise-policy.pdf (accessed 01.05.2021)

48. https://gov.wales/noise-and-soundscape-action-plan-2018-2023-0 (accessed 01.05.2021)

49. https://www.hiddensounds.com.au/installations (accessed 01.05.2021)

50. https://noise-planet.org/noisecapture.html (accessed 01.05.2021)

51. Kang, J., Aletta, F., Margaritis, E. \& Yang, M. A model for implementing soundscape maps in smart cities. Noise Mapping, 5, 46-59 (2018).

52. Aletta, F., Oberman, T., Mitchell, A., Tong, H. \& Kang, J. (2020) Assessing the changing urban sound environment during the COVID-19 lockdown period using short-term acoustic measurements. Noise Mapping, 7, 123-134 (2020).

53. Tong, H., Aletta, F., Mitchell, A., Oberman, T. \& Kang, J. Increases in noise complaints during the COVID-19 lockdown in Spring 2020: a case study in Greater London, UK. Science of the Total Environment, 785, 147213 (2021).

54. Yu, C. \& Kang, J. Soundscape in the sustainable living environment: A cross-cultural comparison between the UK and Taiwan. Science of the Total Environment, 482-483, 501-509 (2014).

55. Torresin S., Albatici, R., Aletta, F., Babich, F., Oberman, T., Siboni, S. \& Kang, J. Indoor soundscape assessment: a principal components model of acoustic perception in residential buildings. Building and Environment, 182, 107152 (2020). 